\title{
Analysis and construction of a competitive endogenous RNA regulatory network of baicalin- induced apoptosis in human osteosarcoma cells
}

\author{
Haifeng Lan \\ Guangzhou medical unversity
}

\section{Haiyan Wang \\ Guangzhou medical unveristy \\ Mi Gao \\ Guangzhou Medical University \\ Jiahuan Zhang \\ Guangzhou Medical University \\ Erkang Yi \\ Guangzhou Medical University}

Chunxiao Liang

Guangzhou Medical University

Xiaoxiao Xiong

Guangzhou Medical University

\section{Xing Chen}

Guangzhou Medical University

Qinghua Wu

Guangzhou Medical University

\section{Ruikun Chen}

Guangzhou Medical University

Wei Hong ( $\sim$ hongwei@gzhmu.edu.cn )

Guangzhou medical unversity https://orcid.org/0000-0003-0265-5863

\section{Research}

Keywords: Baicalin, OS, mRNA, IncRNA, miRNA, ceRNA network

Posted Date: February 5th, 2021

DOI: https://doi.org/10.21203/rs.3.rs-184149/v1

License: (c) (i) This work is licensed under a Creative Commons Attribution 4.0 International License. Read Full License 


\section{Abstract}

Background: Baicalin is an extract from the traditional Chinese herb Scutellaria baicalensis. Recent literature reports have shown the potential of baicalin to treat osteosarcoma (OS). However, the transcriptome-level mechanism of baicalin-mediated antitumor effects in OS has not yet been investigated. The aim of this study was to analyze the competitive endogenous RNA (ceRNA) regulatory network involved in baicalin-induced apoptosis of OS cells.

Methods: In this study, CCK-8 and flow cytometry assays were used to detect the antitumor effects of baicalin on human OS MG63 cells. Furthermore, whole transcriptome sequencing was employed to establish the long noncoding RNA (IncRNA), microRNA (miRNA), and mRNA profiles.

Results: Baicalin could inhibit MG63 cell proliferation and could induce apoptosis. A total of 58 IncRNAs, 31 miRNAs, and 2136 mRNAs in the baicalin-treated MG63 cells were identified as differentially expressed RNAs compared to those in control cells. Of those, 2 IncRNAs, 3 miRNAs, and 18 mRNAs were included in the ceRNA regulatory network. The differentially expressed RNAs were confirmed by using quantitative real-time PCR (qRTPCR). Gene Ontology (GO) and Kyoto Encyclopedia of Genes and Genomes (KEGG) pathway analyses were performed to identify the target genes and signaling pathways of the aberrantly expressed RNAs.

Conclusions: By identifying the ceRNA network, our results provide new information about the possible molecular basis of baicalin, which might be able to be used for OS treatment.

\section{Introduction}

Osteosarcoma (OS) is the most common primary bone tumor with high mortality and poor prognosis ${ }^{[1,2]}$. Although neoadjuvant and postsurgery chemotherapy have improved in recent years, little has changed for patients with metastatic disease, and their long-term survival rate remains at $25-30 \%{ }^{[3]}$. Therefore, there is a great need to explore new diagnostic and treatment strategies to reduce recurrence and improve the survival rate.

Growing evidence indicates the potential of natural compounds to serve as successful anticancer agents ${ }^{[4-6]}$. Baicalin, an important flavonoid, is found in the roots of the Chinese herb Huang-qin (Scutellaria baicalensis Georgi) ${ }^{[7,8]}$. Baicalin exhibits a wide range of pharmacological properties, including antioxidative, antiinflammatory, antiviral, and antiproliferative activities ${ }^{[9-12]}$. Some evidence shows that baicalin has an antitumor effect on OS cells ${ }^{[13-15]}$. It also has beneficial effects when used in the treatment of several cancers. However, the molecular mechanisms underlying the contribution of baicalin to OS treatment remain elusive.

In the last decade, advances in genome-wide analysis of gene expression have revealed that far more of the genome is transcribed than previously anticipated, and the majority of the genome is transcribed into noncoding RNAs (ncRNAs) ${ }^{[16,17]}$. Although most studies on ncRNAs are focused on long noncoding RNAs (IncRNAs, with a length $>200$ nucleotides) and microRNAs (miRNAs, with $<200$ nucleotides), they have recently and rapidly attracted attention. Studies have shown that hundreds of IncRNAs have been discovered, and some IncRNAs have been correlated with tumorigenesis and malignancy transformation in various types of cancers ${ }^{[18-22]}$. However, the potential pathogenesis has not been systematically investigated. In 2011, Salmena et al. ${ }^{[23]}$ 
proposed the competing endogenous RNA (ceRNA) hypothesis, which states that mRNAs, transcribed pseudogenes, and IncRNAs could act as natural miRNA "sponges" and inhibit miRNA function by competing with the binding of one or more microRNA response elements (MREs) in complex and comprehensive regulatory networks, leading to pathogenic conditions. The involvement of the ceRNA regulatory network in tumor initiation and progression has been validated in previous studies. However, the specific ceRNA regulatory network in baicalin-treated human OS cells remains to be elucidated.

In this study, the whole-transcriptome sequencing technique was used to profile the response of MG63 cells to baicalin treatment. Gene Ontology (GO) and Kyoto Encyclopedia of Genes and Genomes (KEGG) analyses were conducted to explore the biological roles and potential pathways of these differentially expressed RNAs. The regulatory ceRNA networks of IncRNA-miRNA-mRNA were constructed based on the sequencing data and bioinformatics analysis. Our findings indicated that in-depth RNA sequencing analysis of ceRNA could be a promising approach to research the anticancer mechanisms of therapeutic agents.

\section{Material And Methods \\ 2.1 Cell culture}

The human OS cell line MG63 was purchased from the American Type Culture Collection (ATCC, VA, USA; CRL$1427 \mathrm{TM}$ ) and cultured in the recommended medium supplemented with $10 \%$ fetal bovine serum (FBS; Gibco, USA) at $37^{\circ} \mathrm{C}$ in a humidified atmosphere with $5 \% \mathrm{CO}_{2}$. Baicalin (purity, $\geq 95 \%$, Sigma-Aldrich, St Louis, MO, USA) was dissolved in dimethyl sulfoxide (DMSO) in a $0.4 \mathrm{mg} / \mathrm{ml}$ stock solution and diluted to different concentrations with culture medium immediately before use. The same volume of DMSO with a final concentration of $0.1 \%$ was used as a negative control.

\subsection{Cell viability assay}

Cells were seeded in a 96-well culture plate at a concentration of 5000 cells/well and treated with baicalin ( 0 , $12.5,25,50,100$, and $200 \mu \mathrm{g} / \mathrm{ml}$ ) for 24,48 , and $72 \mathrm{~h}$. Cells were washed twice with PBS and incubated with $110 \mu \mathrm{L}$ fresh medium containing $10 \mu \mathrm{L}$ CCK-8 solution for an additional $3 \mathrm{~h}$. The plates were analyzed with a Thermo microplate spectrophotometer at a wavelength of $450 \mathrm{~nm}$ (OD450). We calculated the viability with the following formula: cell viability $=($ OD450 of treated groups $/$ OD 450 of control group $) \times 100 \%$.

\subsection{Flow cytometry}

The cells were seeded in a 6 -well plate at a density of $3 \times 10^{5}$ cells/well and then exposed to baicalin $(0,12.5,25$, $50,100$, and $200 \mu \mathrm{g} / \mathrm{ml})$. For apoptosis analysis, cells were harvested and suspended in $500 \mu \mathrm{L}$ of binding buffer containing $5 \mu \mathrm{L}$ of Annexin V-FITC and $5 \mu \mathrm{L}$ of PI, and then the apoptotic cells were analyzed using a FACSCalibur flow cytometer (BD Biosciences).

\subsection{LncRNA isolation and high-throughput sequencing}

To perform IncRNA sequencing analysis, total RNAs were extracted using the miRNeasy Mini Kit (Qiagen). The quality and purity of the extracted RNAs were measured using an Agilent 2100 Bioanalyzer (Agilent Technologies). The IncRNA sequencing library was performed using the Ovation Human FFPE RNA-Seq Library 
System (NUGEN, 0340 - 32), and the sample input was $20 \mathrm{ng}$ of each total RNA. Finally, we profiled the expression of the IncRNA libraries using HiSeq 2500 (Illumina, Inc., San Diego, CA, USA).

\section{5 miRNA isolation and high-throughput sequencing}

To perform miRNA sequencing analysis, total RNA was extracted using the miRNeasy Mini Kit (Qiagen). The quality and purity of the extracted RNAs were measured using an Agilent 2100 Bioanalyzer (Agilent Technologies). The miRNA sequencing library was performed using NEXTflex® Small RNA-Seq Kit v3 (Bio Scientific Corporation, NOVA-5132-05), and the sample input was 20 ng of each total RNA. Finally, we profiled the expression of the miRNA libraries using HiSeq 2500 (Illumina, Inc., San Diego, CA, USA).

\subsection{Analysis of the differentially expressed IncRNAs (DElncRNAs)}

Raw reads were treated with custom Perl script to remove the adapters for read quality control. Then, the read quality was inspected using FastQC and SOAPnuke software, and statistical analyses were run. Paired-end reads were aligned to the human genome version GRCh37 using HISAT2 software. StringTie software was used to assemble the transcripts, and the Perl script was used to screen the known IncRNAs. Then, we obtained the resulting known IncRNAs and used CPC and PFAM software to predict novel IncRNAs. Quantitative analysis of IncRNAs and mRNAs was performed using the R package Ballgown, and the IncRNA target mRNAs were predicted. After obtaining the differential expression of the targeted mRNA reads, we predicted the target and performed enrichment analysis. GO (http://www.geneontology. org) and KEGG (http://www.genome.jp/kegg) analyses were performed for the differentially expressed miRNA (DEmiRNA)-associated genes. A P-value $<0.05$ was considered significant.

\subsection{Analysis of the DEmiRNAs}

Raw reads were treated with custom Perl script to remove the adapters for read quality control. Then, read quality was inspected using FastQC software, and statistical analyses were performed. Paired-end reads were aligned to the human genome version GRCh37 using Bowtie. We used the reads that mapped to the genome to perform more alignment with different software, including NCGB, Rfam, hairpin, etc. to classify the types of reads. Then, we obtained the resultant known miRNAs and used the unknown reads to predict novel miRNAs. All known miRNAs and novel miRNAs were used to calculate expression by performing analysis of variance (AOV) on the transcript per million (TPM) count. After obtaining the differential expression of miRNA reads, we predicted the target and performed enrichment analysis. GO (http://www.geneontology.org) and KEGG (http://www.genome.jp/kegg) analyses were performed for the DEmiRNA-associated genes. A P-value $<0.05$ was considered significant. The top 20 significant pathways of the upregulated miRNAs and downregulated miRNAs were chosen to construct the pathway relation network, which was based on the interaction data in KEGG. The pathway relation network was used to identify the regulatory effect of these pathways. A flow chart of the analysis procedure is illustrated in Fig. 1.

\subsection{Protein-protein interaction (PPI) network}

To understand the interactions of the differentially expressed mRNAs (DEmRNAs), we constructed a PPI network using the Search Tool for the Retrieval of Interacting Genes (STRING, http://string.embl.de/). Combined scores greater than 700 were considered statistically significant. The PPI network was visualized using Cytoscape v 
3.6.1 software. Subsequently, hub gene networks were identified by MCODE v1.5.1. In addition, GO enrichment analysis was performed using STRING to functionally annotate the DEmRNAs in the hub gene network.

\subsection{Construction of the ceRNA network}

A ceRNA network was constructed to discover the ceRNA mechanism based on the differentially expressed RNAs and was established using Cytoscape v 3.6.1 software. First, the IncRNA-miRNA and miRNA-mRNA target relationships were predicted by a target prediction database. miRanda (http://www.microrna.org/microrna/home.do), miRTarBase (http://mirtarbase.mbc.nctu.edu.tw/), and TargetScan (http://www.targetscan.org/) analyses were combined. For a given IncRNA-mRNA pair, both the IncRNA and mRNA were targeted by a common miRNA and negatively coexpressed with this miRNA. Thus, each component of this IncRNA-miRNA-mRNA system was identified as a competing triplet ${ }^{[24]}$.

\subsection{Quantitative real-time PCR (qRT-PCR) validation}

To further improve the ceRNA network reliability, we selected some of the key RNAs in the ceRNA network and used qRT-PCR for validation. RNA samples from MG63 cells treated with different concentrations of baicalin ( 0 and $50 \mu \mathrm{g} / \mathrm{ml}$ ) were collected. Total RNA was extracted using TRIzol reagent (Invitrogen, CA, USA). cDNA was synthesized from $1.0 \mu \mathrm{g}$ of total RNA using the PrimeScript RT ${ }^{\mathrm{TM}}$ Reagent Kit according to the manufacturer's instructions (TaKaRa, Japan). qRT-PCR was carried out using SYBR Premix Ex Taq ${ }^{\text {TM }}$ (TaKaRa) with the Step-One Fast Real-Time PCR system on the CFX Connect ${ }^{\text {TM }}$ Real-Time PCR system (Bio-Rad, USA). For quantitative results, the relative expression levels were calculated using the $2^{-\triangle \Delta C t}$ method. The PCR conditions were 2 min at $95^{\circ} \mathrm{C}$ and 40 cycles of $95^{\circ} \mathrm{C}$ for $5 \mathrm{~s}$ and $60^{\circ} \mathrm{C}$ for $34 \mathrm{~s}$. The primer sequences are shown in Table 1 . The data represent the means of three experiments. 
Table 1

Primer sequences used for qRT-PCR analysis.

\section{Gene name}

primer sequence (5'-3')

\section{IncRNA}

$\begin{array}{lll}\text { ENST00000607286 } & \text { Forward } & \text { GGTTCAGCTCGTGGAAGACA } \\ & \text { Reverse } & \text { TTTAAACGCGCCTACAGGGT } \\ \text { ENST00000449500 } & \text { Forward } & \text { GATGGAACCCTGACCCTGTG } \\ & \text { Reverse } & \text { GACACACATAGACCGCGTGA } \\ \text { GAPDH } & \text { Forward } & \text { CAGCCTCAAGATCATCAGCA } \\ & \text { Reverse } & \text { ACAGTCTTCTGGGTGGCAGT }\end{array}$

miRNA

miR-486-3p

Forward AACAAGTCGGGGCAGCTCA

Reverse GTCGTATCCAGTGCAGGGTCC

RT GTCGTATCCAGTGCAGGGTCCGAGGTATTCGCACTGGATACGACATCCTGT

miR-1908-3p Forward ACCGGCCGCCGGCTCC

Reverse AGTGCAGGGTCCGAGGTATT

RT GTCGTATCCAGTGCAGGGTCCGAGGTATTCGCACTGGATACGACCGGGGC

miR-625-5p Forward AACCGGAGGGGGAAATTC

Reverse GTCGTATCCAGTGCAGGGT

RT GTCGTATCCAGTGCAGGGTCCGAGGTATTCGCACTGGATACGACGGACTA

U6

Forward CTCGCTTCGGCAGCACA

Reverse AACGCTTCACGAATTTGCGT

\section{mRNA}

TREX2 Forward CACCTGATCTCCAGTGACGG

Reverse TGGGGCCAGTTACACAAAGG

B3GNT1 Forward TGCTCCCGGACAAGATATGAG

$\begin{array}{lll} & \text { Reverse } & \text { TGCCATCATCAGGATACCCA } \\ \text { SLC9A7 } & \text { Forward } & \text { TGACTGGTGTTGTGACTGCT } \\ & \text { Reverse } & \text { AACGTGCTCCAGGACATGAG }\end{array}$

ZNF704 Forward TCTTCAGCAAAGCTCCCTGG

Reverse AACGTGCTCCAGGACATGAG 


\begin{tabular}{|c|c|c|}
\hline Gene name & & primer sequence $\left(5^{\prime}-3^{\prime}\right)$ \\
\hline \multirow[t]{2}{*}{ STC2 } & Forward & TGTAGTAGTTGAGCGCAGGC \\
\hline & Reverse & AAGGAGTCGAGCAGGTGTTG \\
\hline \multirow[t]{2}{*}{ WWC3 } & Forward & CCCCGAGAAATTTCAGCCCT \\
\hline & Reverse & CGTGCCACTCCGAACAAAAG \\
\hline \multirow[t]{2}{*}{ WDR35 } & Forward & ATGGAGACATTTGGTGCAACG \\
\hline & Reverse & GAGGCTGCTATCACATGGGT \\
\hline \multirow[t]{2}{*}{ DYRK2 } & Forward & GTTCGTCAGCTTCAGGCTTC \\
\hline & Reverse & CTTACTGCCGCCAATCGTGT \\
\hline \multirow[t]{2}{*}{ KLF13 } & Forward & ACGGGCGAGAAGAAGTTCAG \\
\hline & Reverse & GCATTCCCGGGTGGAAGTTG \\
\hline \multirow[t]{2}{*}{ KDM4B } & Forward & АCСATCACTGTTGCTGGAGG \\
\hline & Reverse & СACTTCTGGATGGCGAGGTT \\
\hline \multirow[t]{2}{*}{ HELZ2 } & Forward & CATCGCAGGTCCCCATCTAC \\
\hline & Reverse & ATTTGGACCCAGAAGAGCCG \\
\hline \multirow[t]{2}{*}{ MAN1A1 } & Forward & AGCCCAGCCTAGGAAAGAGG \\
\hline & Reverse & GGGAGACTCGTCAACTTCGC \\
\hline \multirow[t]{2}{*}{ CPD } & Forward & AGATTGTCTAAAGCATGGCAGT \\
\hline & Reverse & TTCACACTTCCTGTAGCAGTT \\
\hline \multirow[t]{2}{*}{ DPY19L1 } & Forward & CTGGACCACGCTCCTGTTAG \\
\hline & Reverse & TGCGAAAAGCCATCTCССTT \\
\hline \multirow[t]{2}{*}{ PPP1R3G } & Forward & ATCATTGTGTCAGGCAGGGG \\
\hline & Reverse & AAGCCAATTCAAACGGGTGC \\
\hline \multirow[t]{2}{*}{ CAMSAP3 } & Forward & СTTTTCTGGGTGGACACGAC \\
\hline & Reverse & TTGCGGTATCGGATCGAGG \\
\hline \multirow[t]{2}{*}{ ZSWIM5 } & Forward & TTCCCCAGAGTGCCATTCAC \\
\hline & Reverse & GCCAATGTAATTCACGCCCC \\
\hline \multirow[t]{2}{*}{ ANO4 } & Forward & АTCACTTTGCTGGCCTCCTC \\
\hline & Reverse & TTCCTCAGTGCCTTGGTGTC \\
\hline
\end{tabular}

2.11 Statistical analysis 
In this study, data are expressed as the mean \pm SD. All statistical analyses were performed by using SPSS 23.0 (SPSS, Chicago, IL, United States). A P-value $<0.05$ was considered to be significant. For sequencing data, we analyzed DEncRNAs and DEmRNAs using Ballgown (Fu J, 2019) software, with $p<0.05$ and |log2 (fold change)| $>2$ as screening criteria.

\section{Results}

\subsection{Baicalin inhibits human OS cell viability}

To investigate the inhibitory role of baicalin on human OS cell lines (MG63), we employed the CCK-8 assay to determine cell viability. Baicalin was added to the cell medium in a concentration gradient $(0,12.5,25,50,100$ and $200 \mu \mathrm{g} / \mathrm{ml}$ ) for 24,48 , and $72 \mathrm{~h}$. Baicalin inhibited MG63 viability in a dose- and treatment-duration manner (Fig. 2). Our results showed that baicalin significantly inhibits the proliferation of human OS cells.

\subsection{Baicalin induces cell apoptosis in MG63 cells}

Cells were treated with $0,12.5,25,50,100$ or $200 \mu \mathrm{g} / \mathrm{ml}$ baicalin and stained with Annexin V-FITC and PI for apoptosis analysis. As shown in Fig. 3, the percentage of apoptotic cells was significantly increased in a dosedependent manner after baicalin treatment. These results demonstrated baicalin-induced cell apoptosis in MG63 cells.

\subsection{DElncRNAs, DEmiRNAs and DEmRNAs}

In this study, we screened DEIncRNAs, DEmiRNAs, and DEmRNAs using RNA-Seq analysis. Untreated and baicalin-treated MG63 cells were selected for the gene expression assay. The genes with fold-change in expression $>2.0$ and $\mathrm{P}<0.05$ between untreated and baicalin-treated samples were identified to be differentially expressed. In total, our project detected 58 IncRNAs, 31 miRNAs, and 2136 mRNAs. Hierarchical clustering and volcano plots showed DElncRNAs, DEmiRNAs, and DEmRNAs between untreated and baicalin-treated MG63 cells in Fig. 4. There were 58 DElncRNAs (35 upregulated and 23 downregulated), 31 DEmiRNAs (23 upregulated and 8 downregulated), and 2136 DEmRNAs (1023 upregulated and 1113 downregulated) in the baicalin-treated cells compared to those in the untreated cells.

\subsection{GO and KEGG enrichment analyses of DEmRNAs}

To analyze the biological classification of DEmRNAs, GO and KEGG enrichment analyses were performed. The $\mathrm{GO}$ analysis results showed that changes in the biological processes (BPs) of DEGs were mainly enriched in transport, macromolecule metabolic processes, cellular macromolecule metabolic processes and protein metabolic processes. Changes in molecular function (MF) were significantly enriched in nucleic acid binding, cytoskeletal protein binding, and enzyme binding. Changes in the cell component (CC) of DEGs were intensively enriched in intracellular and intracellular parts and intracellular organelles (Fig. 5A). KEGG pathway analysis revealed that the DEmRNAs were mainly enriched in alpha-linolenic acid metabolism, linolenic acid metabolism, biosynthesis of unsaturated fatty acids and ovarian steroidogenesis (Fig. 5B).

\subsection{Construction of a PPI network from the DEmRNAs}


The interrelationship between the DEmRNAs was selected from the STRING database to construct the PPI network. A combined score $>0.4$ was used as the cutoff criterion. As shown in Fig. 6 , a total of 148 nodes and 597 edges were included in this PPI network. The nodes denote DEmRNAs, while the edges denote interactions among the DEmRNAs. Two hub gene networks were identified by MCODE. One included CHEK2, TP53BP1, RECQL4, ATM, SMC4, HMMR, CENPE, CCNB1, ECT2, LIG1, FANCI, and OBFC1 (1), and the other included HSPA8, GNB2L1, PRPF38A, RBCK1, PLK1, GFM1, PSMD12, PPIE, RPL6, PABPC1, EIF4A1, ANAPC13, FBXO22, RPL18, BUD31, SNRPA1, PCF11, RPS17, SMC6 and PSMA2 (2). G0 enrichment analysis showed that the hub genes might function as G2/M transitions of the mitotic cell cycle (1) and spliceosome complex (2).

\subsection{Construction of the ceRNA network}

Many studies have indicated that DElncRNAs could serve as miRNA sponges in organisms. In this study, to better understand the role of those IncRNAs in baicalin-treated MG63 cells, we constructed a ceRNA network based on DEIncRNA-DEmiRNA-DEmRNA interactions by using Cytoscape v 3.6.1 (http://www.cytoscape.org/) software, and the interactions among IncRNAs, miRNAs, and mRNAs were confirmed based on bioinformatics analysis. By using IncRNA as a decoy, miRNA as a center, and mRNA as a target, the IncRNA-miRNA-mRNA regulatory network contained 2 IncRNAs (ENST00000607286 and ENST00000449500), 3 miRNAs (miR-486-3p, miR-1908-3p, and miR-625-5p), and 18 mRNAs (TREX2, B3GNT1, SLC9A7, ZNF704, STC2, WWC3, WDR35, DYRK2, KLF13, KDM4B, HELZ2, MAN1A1, CPD, DPY19L1, PPP1R3G, CAMSAP3, ZSWIM5, and ANO4) (Fig. 7).

\subsection{Expression profile validation}

To validate the accuracy and reliability of the RNA sequencing results, a total of 23 dysregulated IncRNAs and mRNAs in the ceRNA network were selected for qRT-PCR analysis, including 2 IncRNAs, 3 miRNAs, and 18 mRNAs. As shown in Fig. 8, the results from sequencing data were in agreement with those from qRT-PCR in terms of the expression levels of the validated ncRNAs and mRNAs.

\section{Discussion}

OS is the most common malignant bone disease primarily localized to the long bones and is characterized by a high propensity to metastasize ${ }^{[25]}$. Baicalin has been widely used to treat various diseases in traditional Chinese medicine, and it has been reported to exert anticancer functions ${ }^{[26]}$. However, the exact mechanism of its anticancer effects against human OS remains unclear. Moreover, it is vital to identify potential molecular diagnostic markers and/or therapeutic targets to combat human OS.

In recent decades, the complexity of the human genome has been revealed by advanced RNA sequencing analyses, and numerous studies have demonstrated that thousands of IncRNAs are expressed in different kinds of human cancers. Certain IncRNAs behave like oncogenes or tumor suppressors, displaying an important function in cancer initiation, progression, metastasis, and recurrence ${ }^{[27,28]}$. To date, only a few IncRNAs have been experimentally verified, but their roles in regulating gene expression remain to be deciphered. To the best of our knowledge, this is the first study to identify IncRNAs, miRNAs, and mRNAs to reveal regulatory pathways with regard to baicalin-induced apoptosis in MG63 cells. 
Our findings indicated that baicalin could inhibit the proliferation and induce apoptosis of MG63 cells. With $\mid \log 2$ (fold change) $\mid>2$ and p-value $<0.05$ thresholds, a total of 58 IncRNAs, 31 miRNAs, and 2136 mRNAs with significant differential expression were identified in the baicalin-treated MG63 cells compared with expression in the untreated cells. Our study has allowed for a deeper understanding of the antitumor effects in OS. Bioinformatics analysis was performed to explore interactions among the DEmRNAs. The most enriched GO terms of DEmRNAs contained BPs, MFs, and CCs, which included transport, cellular nitrogen compound metabolism, macromolecule metabolic process, intracellular part, intracellular and nucleic acid binding. These GO terms could participate in DNA replication, damage detection, and regulation of the activity of cyclindependent protein serine/threonine kinases. Through KEGG pathway analysis, we detected a number of cancerrelated pathways, including alpha-linolenic acid metabolism, linoleic acid metabolism, biosynthesis of unsaturated fatty acids, the VEGF signaling pathway, and choline metabolism in cancer. Studies have shown that targeting the VEGF-VEGFR pathway seems to be the best approach in hepatic epithelioid hemangioendothelioma ${ }^{[29]}$.

Abnormal choline metabolism continues to be identified in multiple cancers ${ }^{[30]}$. All these above views demonstrate that our ceRNA network reflects vital mechanisms of anticancer effects. Furthermore, we then constructed a PPI network to identify hub DEmRNAs. Proteins that corresponded to genes were used to build the PPI network. A PPI network including 148 proteins and 597 edges was constructed to reveal the relationships among baicalin-treated proteins in MG63 cells. Two core networks of a total of 64 hub genes were identified in this PPI network by using MCODE v1.5.1. GO enrichment analysis showed that the hub genes might function in proteasome-mediated ubiquitin-dependent protein catabolic processes (1) and nuclear-transcribed mRNA catabolic processes (2), indicating that these processes are present in OS. These reports, together with our findings, suggest that these key regulators might play key roles in regulating baicalin-induced apoptosis in human OS cells.

Subsequently, to provide a possible explanation for the baicalin-induced altered RNA expression levels, we established a ceRNA network of IncRNA-miRNA-mRNA according to bioinformatics analysis. The crosstalk between 2 IncRNAs, 3 miRNAs, and 18 mRNAs revealed a complex mechanism in baicalin-treated MG63 cells. The ceRNA hypothesis explains a new mechanism of RNA interaction and provides important clues and theoretical guidance for further understanding the tumorigenesis mechanism ${ }^{[31]}$. We observed that IncRNAs sponge several miRNAs, while miRNAs could regulate more than one mRNA. Based on the ceRNA network, we concluded that the IncRNAs with changed expression were linked with baicalin-treated MG63 cells by sponging the related miRNAs. Such a sponging effect may reflect the regulatory potential of ncRNAs in MG63 cells. In recent years, growing experimental evidence indicates that ceRNA is closely related to the development of cancers $^{[32-34]}$.

LncRNAs, the widest ncRNA subgroup, are RNA molecules of more than 200 bases in length, transcribed by RNA polymerase II, and capped and polyadenylated at their $5^{\prime}$ and $3^{\prime}$ ends, respectively ${ }^{[35]}$. LncRNAs exert both beneficial and detrimental functions by acting at the transcriptional, posttranscriptional, or epigenetic level. In our study, we also identified two DEIncRNAs (ENST00000607286 and ENST00000449500) in the ceRNA networks, which were rarely studied in previous studies. ENST00000607286 is a transcript of two exons with a length of 2204 nucleotides and is located on chromosome 2q13. Our results showed interactions between ENST00000607286 and miR-625-5p and miR-1908-3p. Studies have shown that aberrant expression of miR-

Page $10 / 23$ 
$625-5 p$ in cancers might be a potential risk factor. The mechanisms underlying the antitumor function of miR$625-5 p$ in different cancer types have been demonstrated in previous studies. LINC00958 facilitates cervical cancer cell proliferation and metastasis by sponging miR-625-5p to upregulate LRRC8E expression, which provides a novel biomarker for experimenters to discover better treatments for cervical cancer patients ${ }^{[36]}$. LINC009581 elevated the expression of CPSF7 by acting as a miR-625-5p sponge, which accelerated the development and progression of lung adenocarcinoma, thereby demonstrating that LINC009581 might be utilized as a promising therapeutic target for lung adenocarcinoma ${ }^{[37]}$. LINC00511 is a tumor promoter that sponges miR-625-5p by targeting NFIX in gastric cancer cells, which could be considered a brand new target for gastric cancer treatment ${ }^{[38]}$. Novel hypoxia-associated circDENND2A enhances the migration and invasion of glioma cells by directly sponging miR-625-5p ${ }^{[39]}$.

In addition, ENST00000449500 is located on chromosome 20 with a total length of 801 bp and two exons. Our results also showed intimate interactions between ENST00000449500 and miRNAs, such as miR-486-3p and miR-1908-3p. Unlike IncRNAs, miRNAs, a group of endogenous, evolutionarily conserved nonprotein-coding RNA molecules with a typical length of 20-24 nucleotides, essentially regulate gene expression via posttranscriptional regulation. ceRNAs can competitively bind to MREs, revealing that miRNAs are at the center of ceRNA networks. It has been reported that dysregulated miRNAs play various roles in the initiation, progression, invasiveness, and metastasis of tumors ${ }^{[40]}$. miRNAs are involved in multiple roles during carcinogenesis. Among them, miR-486-3p has been shown to exert a regulatory role in tumor progression. Chou et al. ${ }^{[41]}$ demonstrated that miRNA-486-3p functions as a tumor suppressor in oral cancer by targeting DDR1. Another study on cervical cancer suggested that c-Myc could upregulate IncRNA-PVT1 expression, which subsequently releases the inhibition of ECM1 by sponging miR-486-3p, thus enhancing the proliferation and viability of cervical cancer cells. The c-Myc/IncRNA-PVT1/miR-486-3p/ECM1 axis might serve as a new target for more efficient diagnosis and treatment of cervical cancer ${ }^{[42]}$. In laryngeal squamous cell carcinoma (LSCC), circFLNA functions in LSCC migration by sponging miR-4863p which downregulates FLNA protein expression. Targeting the circFLNA/miR4863p/FLAN axis provides a potential therapeutic target for aggressive LSCC $^{[43]}$. Moreover, miR-486-3p may serve as a biomarker for the detection of oral tongue squamous cell carcinoma[44]. To date, no study has reported any association of miR-1908-3p with cancer. Moreover, our studies have reported that the apoptosis-related miRNAs miR-130a, miR-222, miR-195, miR-29c, miR-92a-1, and miR-216a were aberrantly expressed in baicalin-induced apoptosis in MG63 cells. Interestingly, among them, miR-195, miR-29c, and miR-92a-1 were reported in a previous study of osteosarcoma ${ }^{[45-47]}$, indicating the high degree of confidence in our sequencing results. This is the first study to show aberrant expression of ENST00000449500 and ENST00000607286 in MG63 cells and indicates a potential prognostic role of this 2-IncRNA signature in MG63 cells. In addition, bioinformatics-based investigations of IncRNAs will be helpful in future experimental studies.

Eventually, the selected RNAs in the ceRNA network were verified by qRT-PCR to confirm the reliability and validity of the above bioinformatics results. The 18 verified RNA expression levels were in line with the sequencing results, indicating the high degree of confidence of this network. We verified the independent RNAs, and the results supported our findings and provided a better understanding of IncRNA-related ceRNAs and their important role in baicalin-induced apoptosis of MG63 cells. 
ENST00000449500 is also called IncRNA melanoma highly expressed noncoding RNA (MHENCR); Chen et al ${ }^{[48]}$ found that MHENCR promotes melanoma progression by regulating the miR-425/489-mediated PI3K-Akt pathway. According to our research, IncRNA MHENCR might affect the OS process by regulating miR-1908$3 p / m i R-468-3 p$, indicating a new mechanism of MHENCR in OS. Another IncRNA, ENST00000607286, named SLC9A3-AS1 on NCBI, was one of thirteen transcripts. Bai et al ${ }^{[49]}$ found that SLC9A3-AS1 was highly expressed in the peripheral blood of lung cancer patients, but there is no research on its function so far.

To our knowledge, our work is the first expression profile that analyzes and validates the ceRNA-mediated mechanism to signify how baicalin exerts its antitumor effects in OS. Although the findings of our study have important clinical implications, the limitations must also be noted. First, a comparative analysis of the two groups of ceRNA networks showed only two DElncRNAs and three DEmiRNAs. Second, ceRNA binding experiments also need to be further investigated. Further experiments are needed to support the identification of functional roles.

\section{Conclusions}

In summary, the present study demonstrated that baicalin can reduce cell viability and induce cell apoptosis in MG63 cells. DEncRNAs, DEmiRNAs, and DEmRNAs were identified, and a functional IncRNA-miRNA-mRNA ceRNA regulatory network for MG63 cells without and with baicalin intervention was successfully constructed. Bioinformatics analysis may provide a better understanding of the potential roles of RNAs in human OS cells treated with baicalin, and these RNAs might serve as prognostic biomarkers and therapeutic targets. Two DElncRNAs (ENST00000607286 and ENST00000449500), 3 DEmiRNAs (miR-486-3p, miR-1908-3p, and miR625-5p) and 18 DEmRNAs (TREX2, B3GNT1, SLC9A7, ZNF704, STC2, WWC3, WDR35, DYRK2, KLF13, KDM4B, HELZ2, MAN1A1, CPD, DPY19L1, PPP1R3G, CAMSAP3, ZSWIM5, and ANO4) were identified in the ceRNA network to be closely associated with OS pathogenesis. The ceRNA regulatory network might illuminate the inner molecular mechanism involved in the tumorigenesis and progression of OS. Moreover, the corresponding roles and molecular mechanisms of these ncRNAs and mRNAs need to be further elucidated.

\section{Abbreviations}

Baicalin, OS, ceRNA network

\section{Declarations}

\section{Ethics approval and consent to participate}

Not applicable

\section{Consent to publish}

Consent

\section{Availability of data and materials}

Not applicable 


\section{Competing interests}

The authors declare that there are no conflicts of interest.

\section{Funding}

This work was supported by Ph.D. Initiation Project of the Third Affiliated Hospital of Guangzhou Medical University (grant number 2018B14), Characteristic Innovation Projects of Universities in Guangdong Province (2019KTSCX139), Open Project of the State Key Laboratory of Respiratory Disease (SKLRD-Z-202103), National Natural Science Foundation of China (82000045), 2019 Laboratory Opening Project of Guangzhou Medical University (201910570001).

\section{Authors' Contributions}

Not applicable

\section{Acknowledgements}

Not applicable

\section{References}

1. Ottaviani G, Jaffe N. The epidemiology of osteosarcoma. Cancer Treat Res 2009, 152: 3-13.

2. Song K, Song J, Lin K, Chen F, Ma X, Jiang J, Li F. Survival analysis of patients with metastatic osteosarcoma: a Surveillance, Epidemiology, and End Results population-based study. Int Orthop 2019, 43(8): 1983-1991.

3. Qiu S, Tao L, Zhu Y. Marital Status and Survival in Osteosarcoma Patients: An Analysis of the Surveillance, Epidemiology, and End Results (SEER) Database. Med Sci Monit 2019, 25: 8190-8203.

4. Francisco Fernandez M, Charfi C, Piloto-Ferrer J, Lidia Gonzalez M, Lamy S, Annabi B. Targeting Ovarian Cancer Cell Cytotoxic Drug Resistance Phenotype with Xanthium strumarium L. Extract. Evid Based Complement Alternat Med 2019, 2019: 6073019.

5. Peng F, Xie X, Peng C. Chinese Herbal Medicine-Based Cancer Therapy: Novel Anticancer Agents Targeting MicroRNAs to Regulate Tumor Growth and Metastasis. Am J Chin Med 2019, 47(8): 1711-1735.

6. Xiang Y, Zhang Q, Wei S, Huang C, Li Z, Gao Y. Paeoniflorin: a monoterpene glycoside from plants of Paeoniaceae family with diverse anticancer activities. J Pharm Pharmacol 2019.

7. Moore OA, Gao Y, Chen AY, Brittain R, Chen YC. The Extraction, Anticancer Effect, Bioavailability, and Nanotechnology of Baicalin. J Nutr Med Diet Care 2016, 2(1).

8. Martinez Medina JJ, Naso LG, Perez AL, Rizzi A, Ferrer EG, Williams PAM. Antioxidant and anticancer effects and bioavailability studies of the flavonoid baicalin and its oxidovanadium(IV) complex. J Inorg Biochem 2017, 166: 150-161.

9. Jia Y, Xu R, Hu Y, Zhu T, Ma T, Wu H, Hu L. Anti-NDV activity of baicalin from a traditional Chinese medicine in vitro. J Vet Med Sci 2016, 78(5): 819-824.

10. Dou J, Wang Z, Ma L, Peng B, Mao K, Li C, Su M, Zhou C, Peng G. Baicalein and baicalin inhibit colon cancer using two distinct fashions of apoptosis and senescence. Oncotarget 2018, 9(28): 20089-20102. 
11. Gong L, Zhu J. Baicalin alleviates oxidative stress damage in trabecular meshwork cells in vitro. Naunyn Schmiedebergs Arch Pharmacol 2018, 391(1): 51-58.

12. Yu F, Xu N, Zhou Y, Li B, Li M, Wang Q, Yang X, Ge X, Zhang F, Ren X. Anti-inflammatory effect of paeoniflorin combined with baicalin in oral inflammatory diseases. Oral Dis 2019, 25(8): 1945-1953.

13. Wang Y, Wang H, Zhou R, Zhong W, Lu S, Ma Z, Chai Y. Baicalin inhibits human osteosarcoma cells invasion, metastasis, and anoikis resistance by suppressing the transforming growth factor-beta1-induced epithelial-to-mesenchymal transition. Anti-cancer drugs 2017, 28(6): 581-587.

14. Wan D, Ouyang H. Baicalin induces apoptosis in human osteosarcoma cell through ROS-mediated mitochondrial pathway. Nat Prod Res 2018, 32(16): 1996-2000.

15. Liu Y, Hong Z, Chen P, Wang J, Zhou Y, Huang J. Baicalin inhibits growth and induces apoptosis of human osteosarcoma cells by suppressing the AKT pathway. Oncology letters 2019, 18(3): 3188-3194.

16. Arrigoni A, Ranzani V, Rossetti G, Panzeri I, Abrignani S, Bonnal RJ, Pagani M. Analysis RNA-seq and Noncoding RNA. Methods Mol Biol 2016, 1480: 125-135.

17. Akhade VS, Pal D, Kanduri C. Long Noncoding RNA: Genome Organization and Mechanism of Action. Advances in experimental medicine and biology 2017, 1008: 47-74.

18. Fan LY, Shi KY, Xu D, Ren LP, Yang P, Zhang L, Wang F, Shao GL. LncRNA GIHCG regulates microRNA-1281 and promotes malignant progression of breast cancer. Eur Rev Med Pharmacol Sci 2019, 23(24): 1084210850.

19. Zhao M, Xin XF, Zhang JY, Dai W, Lv TF, Song Y. LncRNA GMDS-AS1 inhibits lung adenocarcinoma development by regulating miR-96-5p/CYLD signaling. Cancer Med 2019.

20. Bhan A, Soleimani M, Mandal SS. Long Noncoding RNA and Cancer: A New Paradigm. Cancer research 2017, 77(15): 3965-3981.

21. Li J, Li Z, Zheng W, Li X, Wang Z, Cui Y, Jiang X. LncRNA-ATB: An indispensable cancer-related long noncoding RNA. Cell Prolif 2017, 50(6).

22. Ma S, Long T, Huang WJM. Noncoding RNAs in Inflammation and Colorectal Cancer. RNA biology 2019.

23. Salmena L, Poliseno L, Tay Y, Kats L, Pandolfi PP. A ceRNA hypothesis: the Rosetta Stone of a hidden RNA language? Cell 2011, 146(3): 353-358.

24. Hansen TB, Jensen TI, Clausen BH, Bramsen JB, Finsen B, Damgaard CK, Kjems J. Natural RNA circles function as efficient microRNA sponges. Nature 2013, 495(7441): 384-388.

25. He C, Liu C, Wang L, Sun Y, Jiang Y, Hao Y. Histone methyltransferase NSD2 regulates apoptosis and chemosensitivity in osteosarcoma. Cell death \& disease 2019, 10(2): 65.

26. Ma W, Liu X, Du W. Baicalin induces apoptosis in SW480 cells through downregulation of the SP1 transcription factor. Anti-cancer drugs 2019, 30(2): 153-158.

27. Begolli R, Sideris N, Giakountis A. LncRNAs as Chromatin Regulators in Cancer: From Molecular Function to Clinical Potential. Cancers (Basel) 2019, 11(10).

28. Mishra K, Kanduri C. Understanding Long Noncoding RNA and Chromatin Interactions: What We Know So Far. Noncoding RNA 2019, 5(4).

29. Telli TA, Okten IN, Tuylu TB, Demircan NC, Arikan R, Alan O, Ercelep O, Ones T, Yildirim AT, Dane F, Yumuk PF. VEGF-VEGFR pathway seems to be the best target in hepatic epithelioid hemangioendothelioma: A case 
series with review of the literature. Curr Probl Cancer 2020: 100568.

30. Glunde K, Penet MF, Jiang L, Jacobs MA, Bhujwalla ZM. Choline metabolism-based molecular diagnosis of cancer: an update. Expert Rev Mol Diagn 2015, 15(6): 735-747.

31. Yao Y, Zhang T, Qi L, Liu R, Liu G, Wang J, Song Q, Sun C. Comprehensive analysis of prognostic biomarkers in lung adenocarcinoma based on aberrant IncRNA-miRNA-mRNA networks and Cox regression models. Bioscience reports 2020, 40(1).

32. Fan CN, Ma L, Liu N. Systematic analysis of IncRNA-miRNA-mRNA competing endogenous RNA network identifies four-IncRNA signature as a prognostic biomarker for breast cancer. J Transl Med 2018, $16(1): 264$.

33. Tang F, Lu Z, Wang J, Li Z, Wu W, Duan H, He Z. Competitive endogenous RNA (ceRNA) regulation network of IncRNAs, miRNAs, and mRNAs in Wilms tumour. BMC Med Genomics 2019, 12(1): 194.

34. Wang L, Cho KB, Li Y, Tao G, Xie Z, Guo B. Long Noncoding RNA (IncRNA)-Mediated Competing Endogenous RNA Networks Provide Novel Potential Biomarkers and Therapeutic Targets for Colorectal Cancer. International journal of molecular sciences 2019, 20(22).

35. Kazimierczyk M, Kasprowicz MK, Kasprzyk ME, Wrzesinski J. Human Long Noncoding RNA Interactome: Detection, Characterization and Function. International journal of molecular sciences 2020, 21(3).

36. Wang L, Zhong Y, Yang B, Zhu Y, Zhu X, Xia Z, Xu J, Xu L. LINC00958 facilitates cervical cancer cell proliferation and metastasis by sponging miR-625-5p to upregulate LRRC8E expression. Journal of cellular biochemistry 2020, 121(3): 2500-2509.

37. Yang L, Li L, Zhou Z, Liu Y, Sun J, Zhang X, Pan H, Liu S. SP1 induced long non-coding RNA LINC00958 overexpression facilitate cell proliferation, migration and invasion in lung adenocarcinoma via mediating miR-625-5p/CPSF7 axis. Cancer Cell Int 2020, 20: 24.

38. Chen Z, Wu H, Zhang Z, Li G, Liu B. LINC00511 accelerated the process of gastric cancer by targeting miR625-5p/NFIX axis. Cancer Cell Int 2019, 19: 351.

39. Su H, Zou D, Sun Y, Dai Y. Hypoxia-associated circDENND2A promotes glioma aggressiveness by sponging miR-625-5p. Cell Mol Biol Lett 2019, 24: 24.

40. Iqbal MA, Arora S, Prakasam G, Calin GA, Syed MA. MicroRNA in lung cancer: role, mechanisms, pathways and therapeutic relevance. Mol Aspects Med 2019, 70: 3-20.

41. Chou ST, Peng HY, Mo KC, Hsu YM, Wu GH, Hsiao JR, Lin SF, Wang HD, Shiah SG. MicroRNA-486-3p functions as a tumor suppressor in oral cancer by targeting DDR1. J Exp Clin Cancer Res 2019, 38(1): 281.

42. Wang C, Zou H, Chen A, Yang H, Yu X, Yu X, Wang Y. C-Myc-activated long non-coding RNA PVT1 enhances the proliferation of cervical cancer cells by sponging miR-486-3p. J Biochem 2020.

43. Wang JX, Liu Y, Jia XJ, Liu SX, Dong JH, Ren XM, Xu O, Zhang HZ, Duan HJ, Shan CG. Upregulation of circFLNA contributes to laryngeal squamous cell carcinoma migration by circFLNA-miR-486-3p-FLNA axis. Cancer Cell Int 2019, 19: 196.

44. Chen Z, Yu T, Cabay RJ, Jin Y, Mahjabeen I, Luan X, Huang L, Dai Y, Zhou X. miR-486-3p, miR-139-5p, and miR-21 as Biomarkers for the Detection of Oral Tongue Squamous Cell Carcinoma. Biomark Cancer 2017, 9: 1-8.

45. Xu N, Xu J, Zuo Z, Liu Y, Yan F, Han C. Downregulation of IncRNA SNHG12 reversed IGF1R-induced osteosarcoma metastasis and proliferation by targeting miR-195-5p. Gene 2020, 726: 144145. 
46. Zhang Z, Liu J, Wu Y, Zhao X, Hao Y, Wang X, Xue C, Wang Y, Zhang R, Zhang X. Long Noncoding RNA SERTAD2-3 Inhibits Osteosarcoma Proliferation and Migration by Competitively Binding miR-29c. Genet Test Mol Biomarkers 2020, 24(2): 67-72.

47. Yu H, Song H, Liu L, Hu S, Liao Y, Li G, Xiao X, Chen X, He S. MiR-92a modulates proliferation, apoptosis, migration, and invasion of osteosarcoma cell lines by targeting Dickkopf-related protein 3. Bioscience reports 2019, 39(4).

48. Chen X, Dong H, Liu S, Yu L, Yan D, Yao X, Sun W, Han D, Gao G. Long noncoding RNA MHENCR promotes melanoma progression via regulating miR-425/489-mediated PI3K-Akt pathway. Am J Transl Res 2017, 9(1): 90-102.

49. Bai Y, Qu Y, Wu Z, Ren Y, Cheng Z, Lu Y, Hu J, Lou J, Zhao J, Chen C, Mao H. Absolute quantification and analysis of extracellular vesicle IncRNAs from the peripheral blood of patients with lung cancer based on multi-colour fluorescence chip-based digital PCR. Biosens Bioelectron 2019, 142: 111523.

\section{Figures}




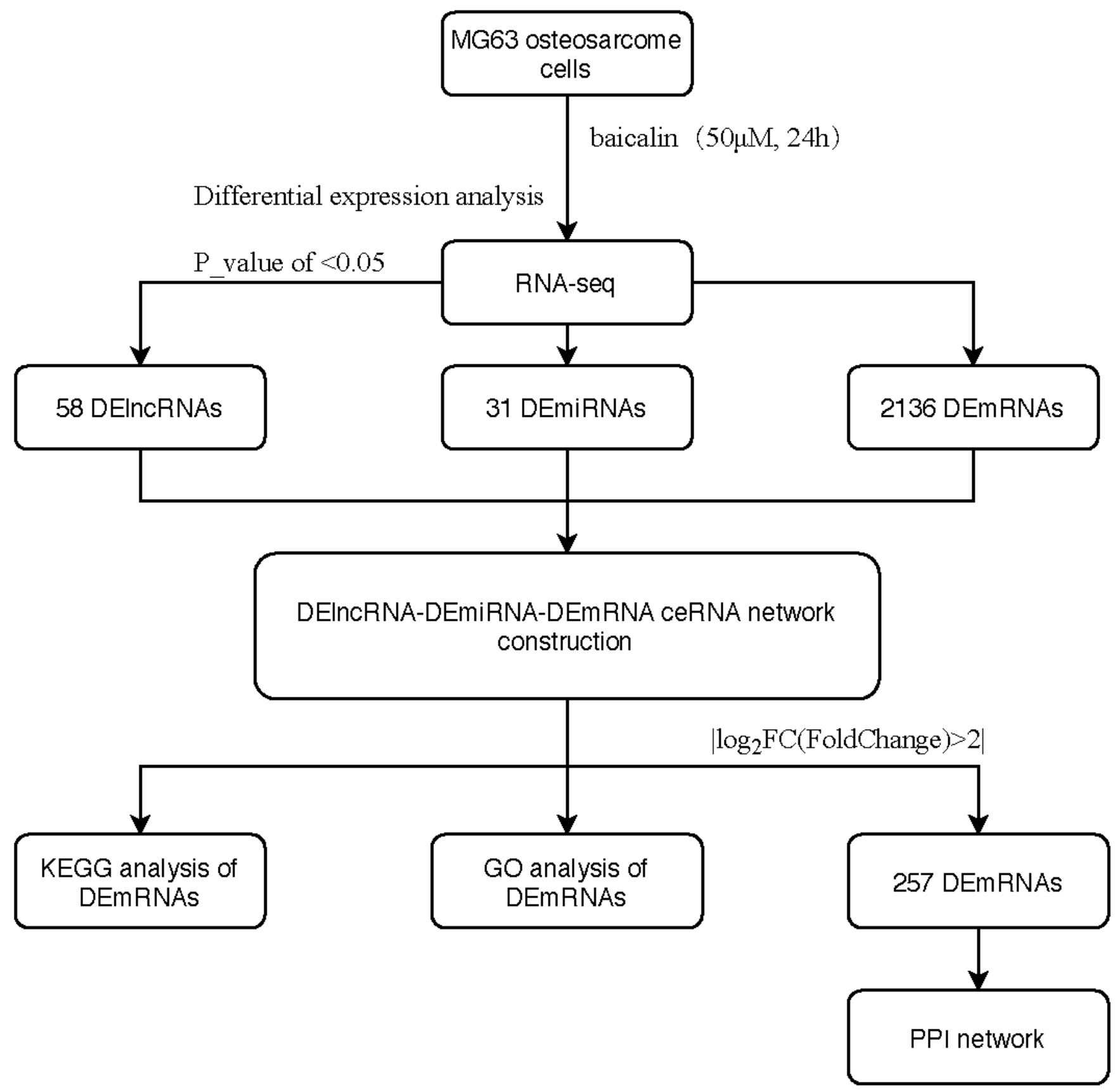

Figure 1

The flow chart of the analytical procedure used in this study. 


\section{MG63}

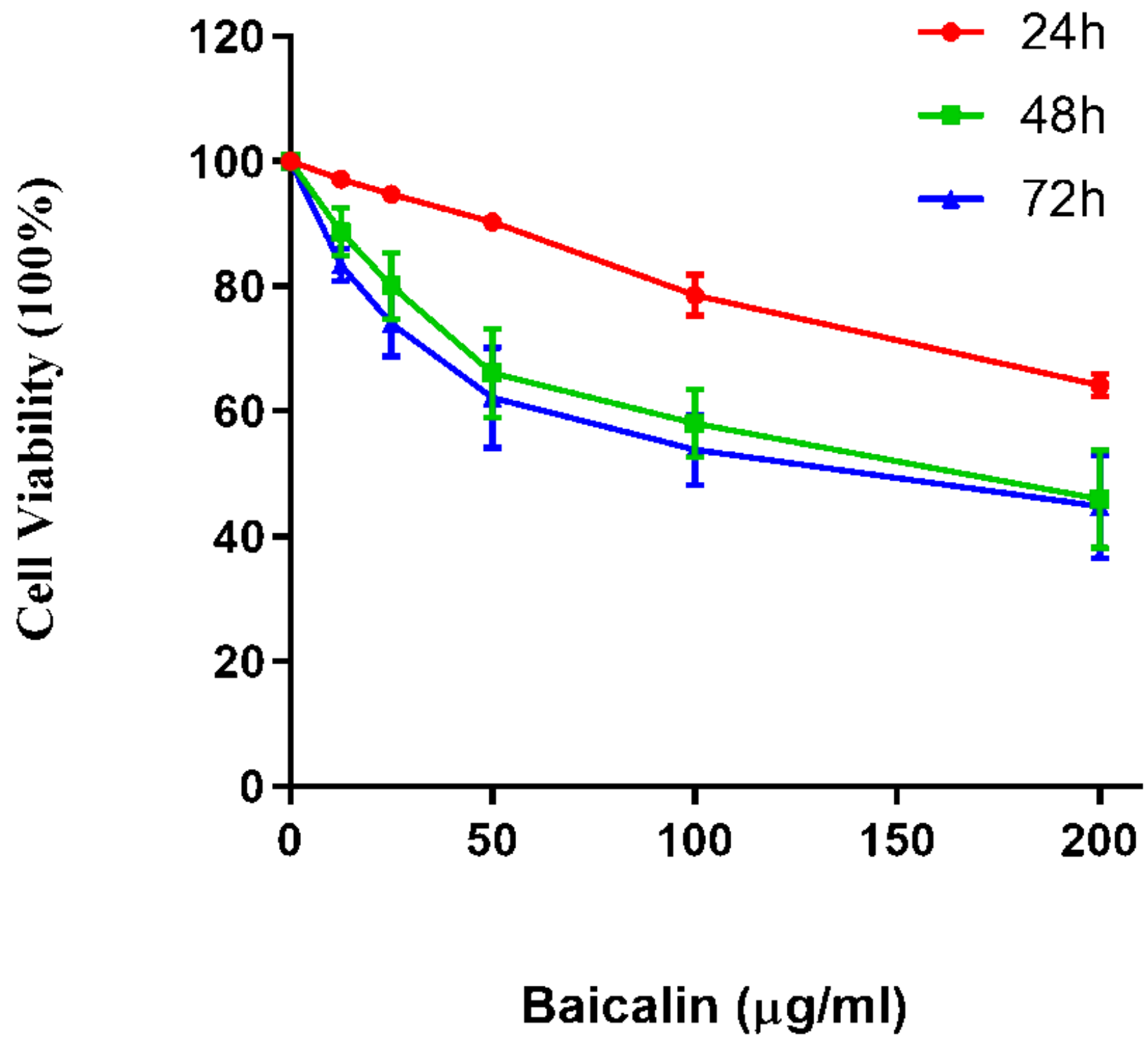

Figure 2

Effect of baicalin treatment on the viability and survival of MG63 cells. MG63 cells were treated with $0,12.5,25$, 50,100 and $200 \mu \mathrm{g} / \mathrm{ml}$ baicalin for 24,48 or $72 \mathrm{~h}$. A cell counting kit-8 (CCK8) was used to measure the cell viability of the treated cells relative to that of the untreated MG63 cells. The data are presented as the mean \pm SD of three independent experiments. 
A

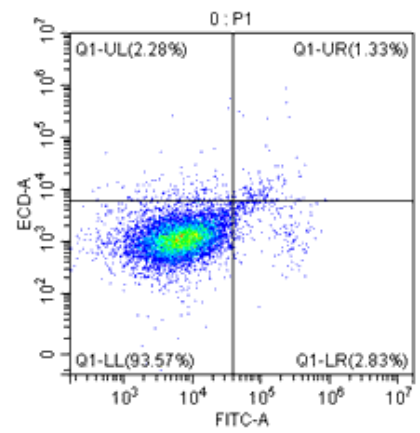

$\mathrm{E}$

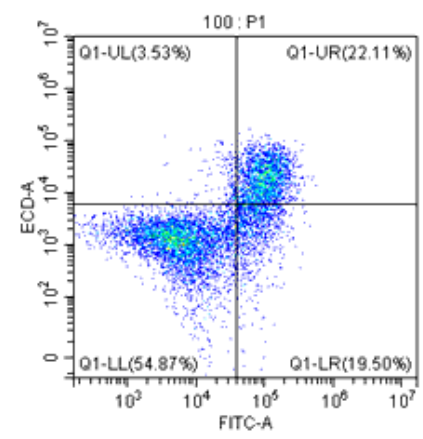

$\mathrm{B}$

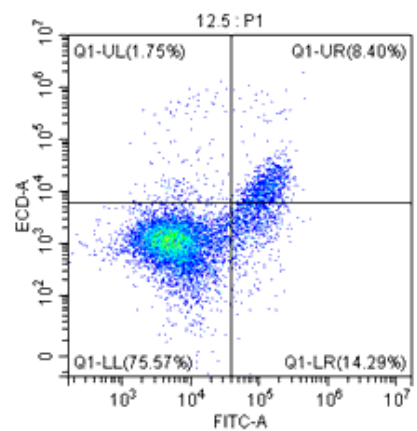

$\mathrm{F}$

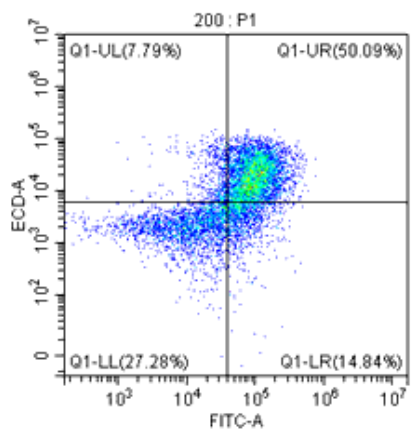

$\mathrm{C}$

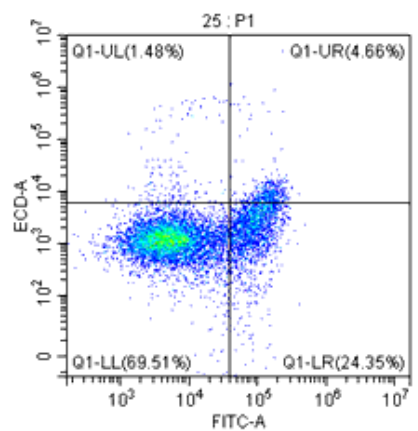

G

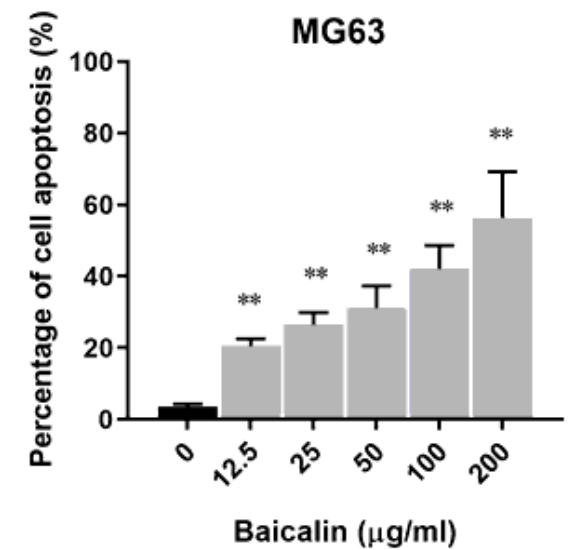

Baicalin $(\mu \mathrm{g} / \mathrm{ml})$

$\mathrm{D}$

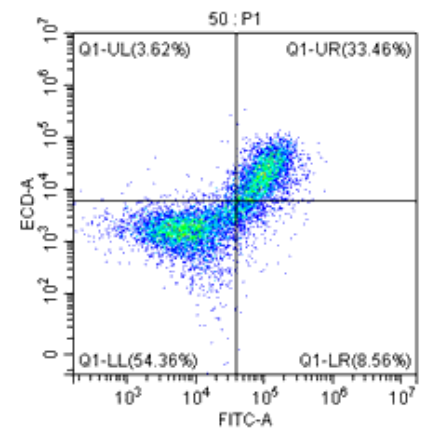

Figure 3

Effect of baicalin treatment on apoptosis of MG63 cells. Cells were treated with $0,12.5,25,50,100$ and 200 $\mu \mathrm{g} / \mathrm{ml}$ baicalin. The percentage of apoptotic cells was measured using Annexin V PI staining and flow cytometry analysis. The data are presented as the mean \pm SD of three independent experiments, ${ }^{*} \mathrm{P}<0.01$.
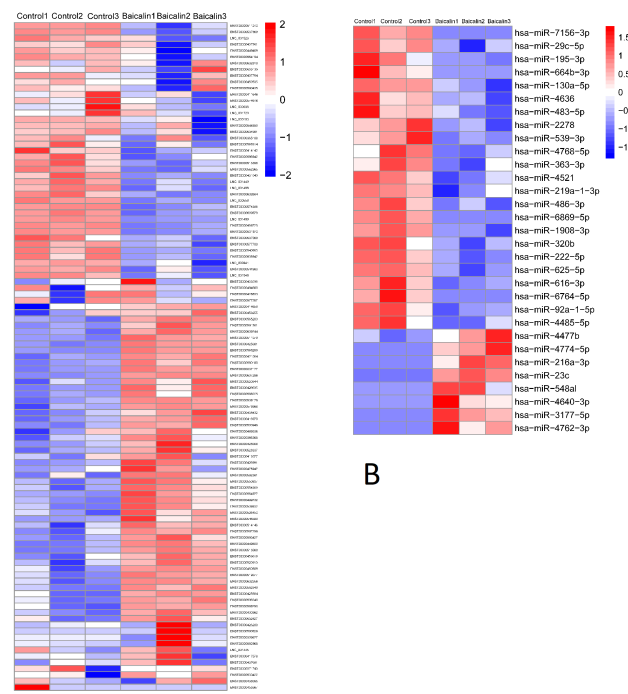

B

A

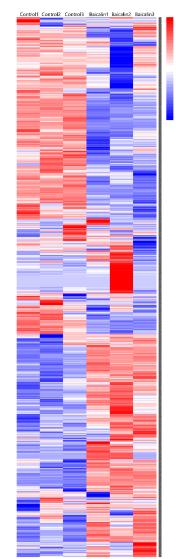

C
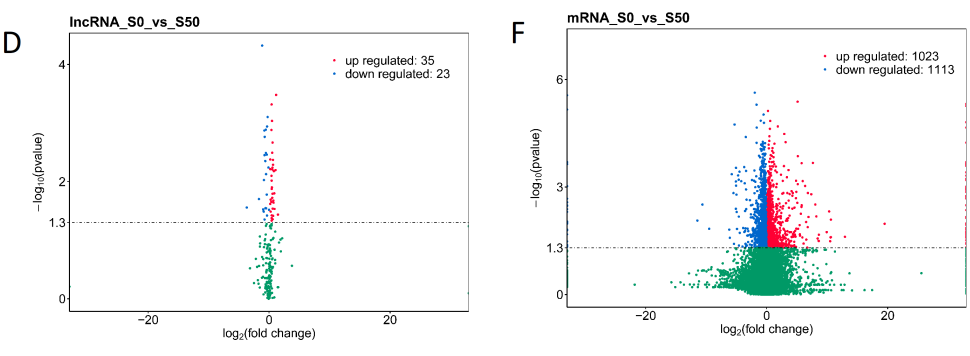

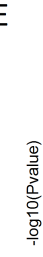

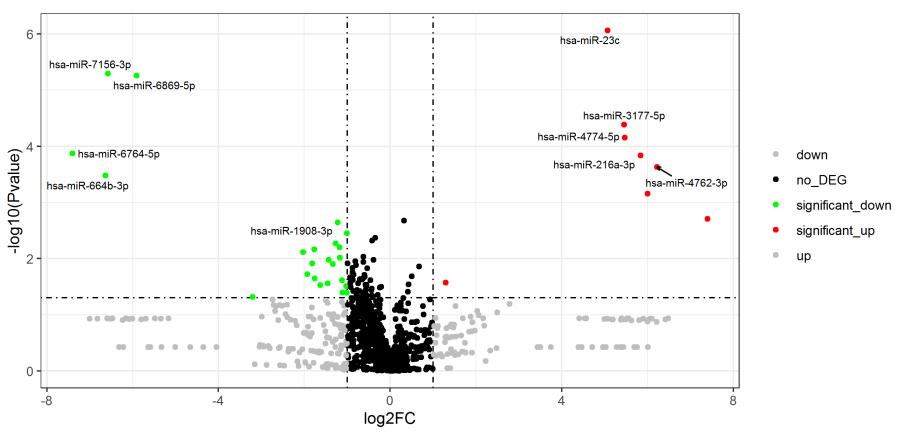


Figure 4

Identification of DElncRNAs, DEmiRNAs and DEmRNAs in gastric cancer. Hierarchical clustering of DElncRNAs (A), DEmiRNAs (B), and DEmRNAs (C) between untreated and baicalin-untreated MG63 cells. Volcano plot showing DElncRNAs (D), DEmiRNAs (E), and DEmRNAs (F) between untreated and baicalin-treated MG63 cells.
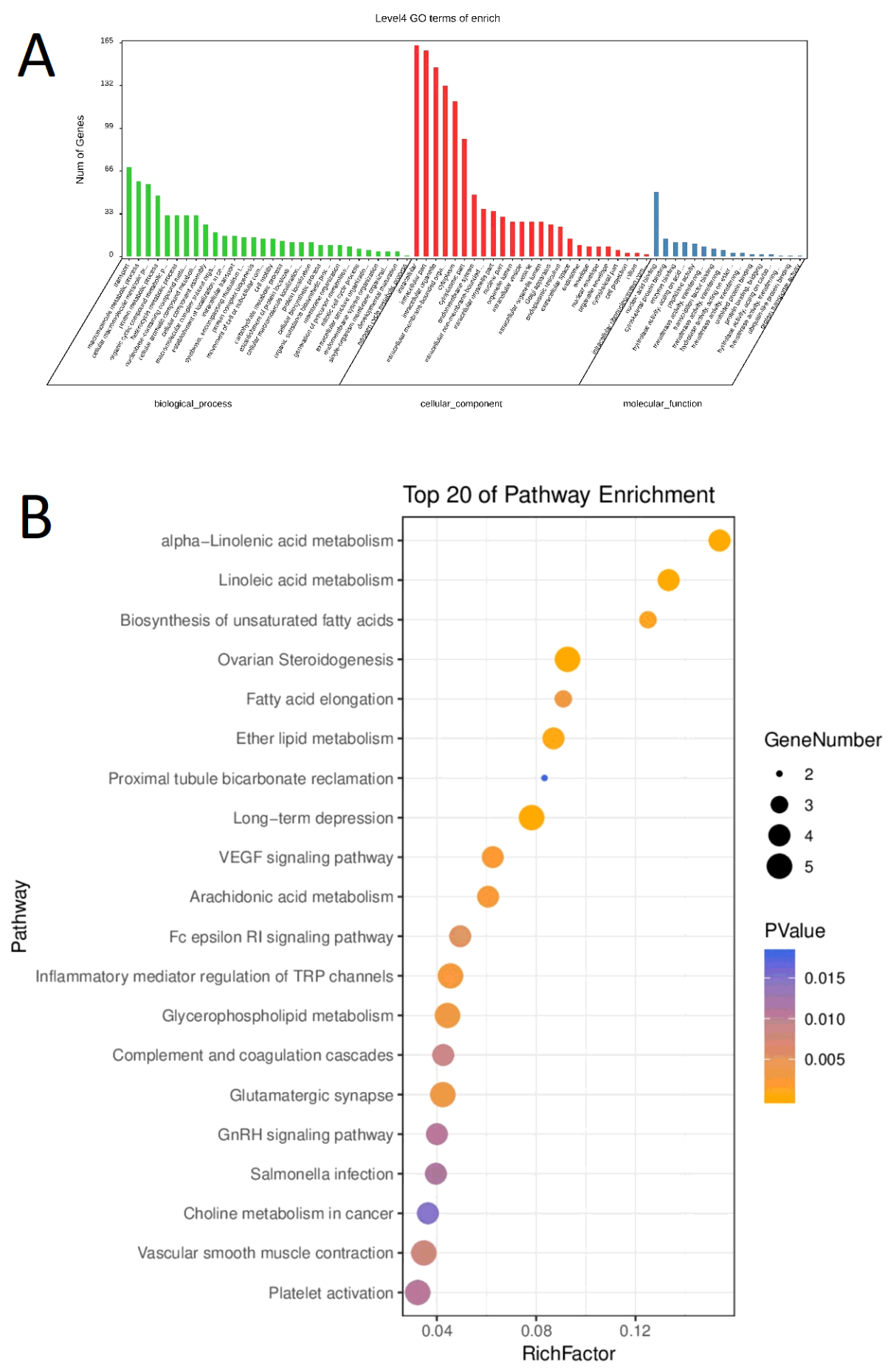

Figure 5

GO and KEGG pathway enrichment analysis of target genes. (A) GO enrichment of mRNAs interacting with IncRNAs. Points of different shapes represent BPs, CCs and MFs from the GO analysis, and the bar plot shows the number of genes enriched in the GO function. (B) Histogram of KEGG pathway enrichment in baicalin- 
treated MG63 cells. The size of the dots represents the number of genes annotated in the pathway, and the color of the dots represents the corrected $p$-value of the hypergeometric test.
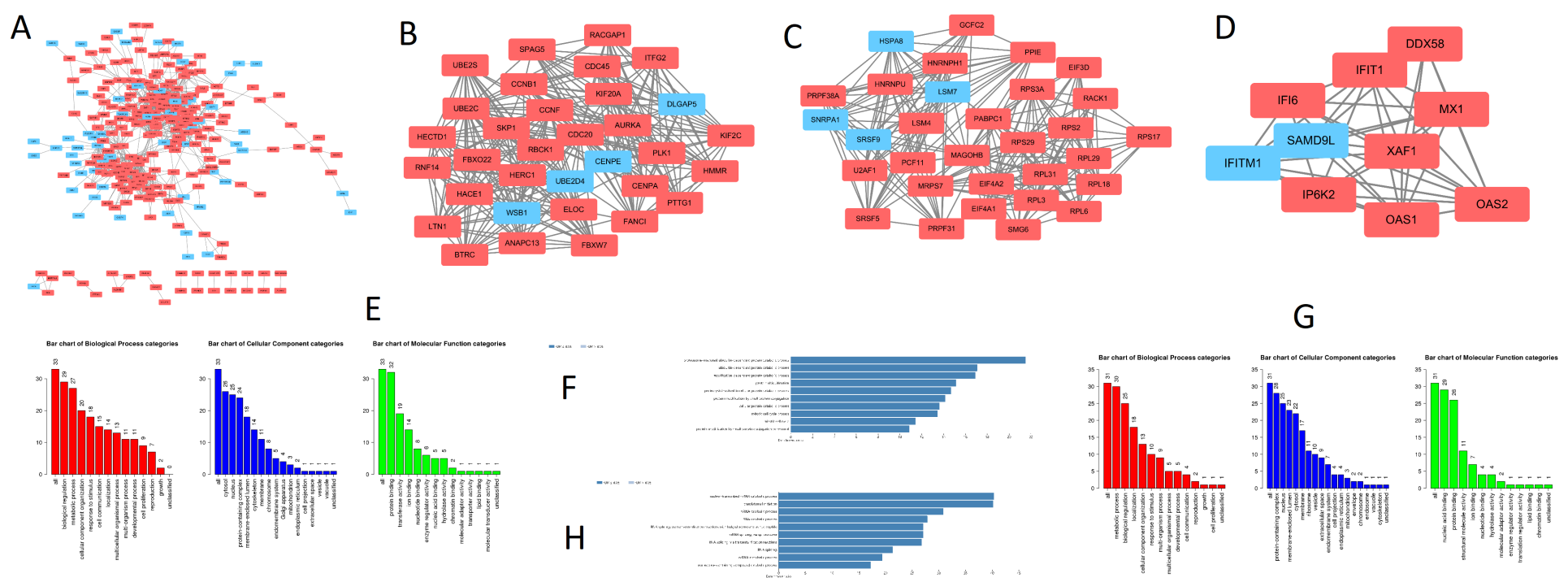

\section{Figure 6}

The PPIs of RNA. (A) PPI network. The value of the centrality degree is marked by different node sizes. Up- or downregulation of genes according to baicalin-untreated MG63 cells vs. control comparison are filled with red or blue color, respectively. (B-D) Three hub gene networks were identified by MCODE. (E-F) GO and KEGG pathway enrichment analyses of the hub gene network B. (G-H) GO and KEGG pathway enrichment analyses of the hub gene network $\mathrm{C}$. 


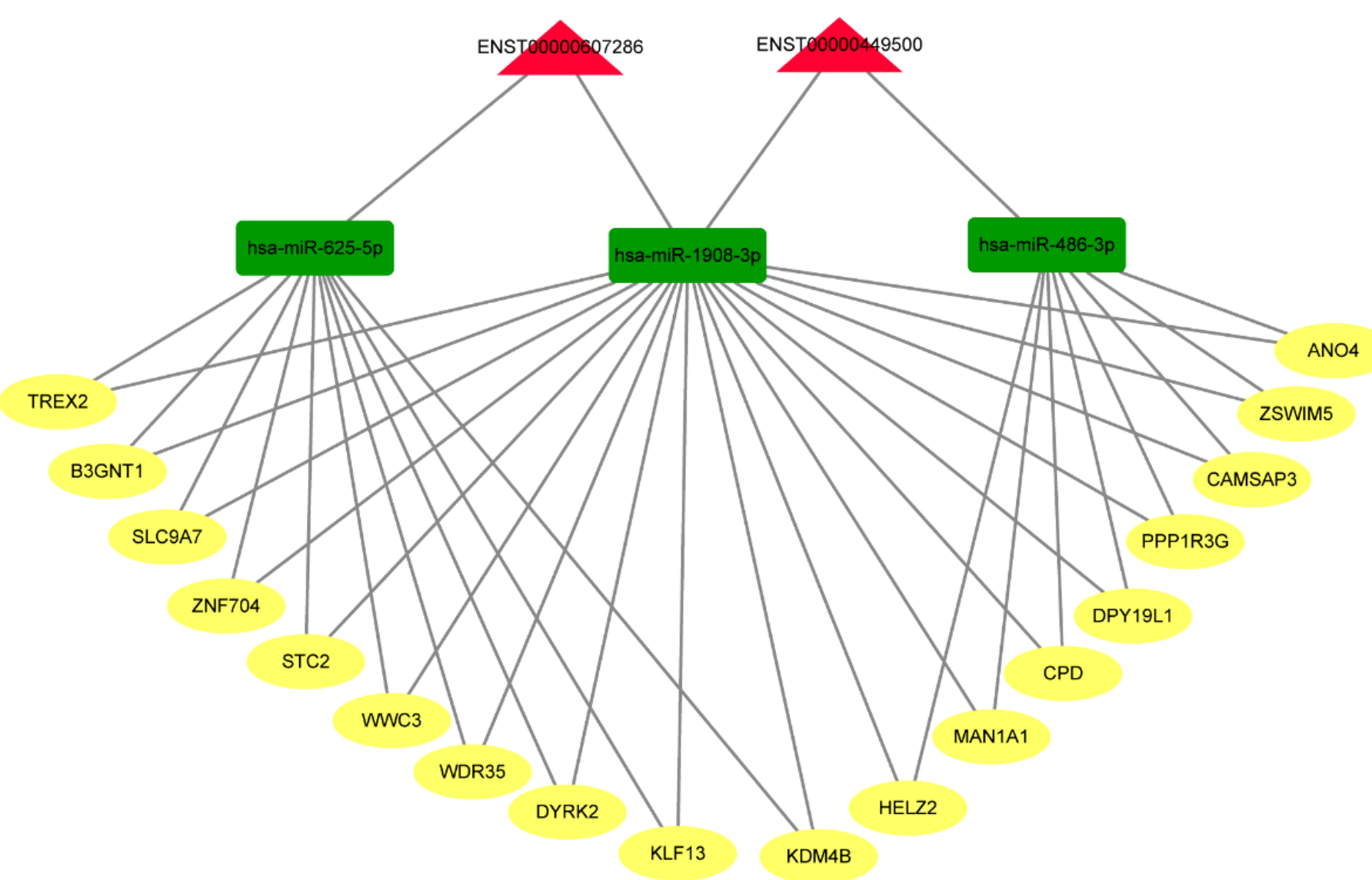

Figure 7

CeRNA network analysis of DEIncRNAs, DEmiRNAs and DEmRNAs in baicalin-treated MG63 cells compared to those in the control group. In the network, red triangles represent IncRNAs, green rectangles represent miRNAs and yellow circles represent mRNAs. 

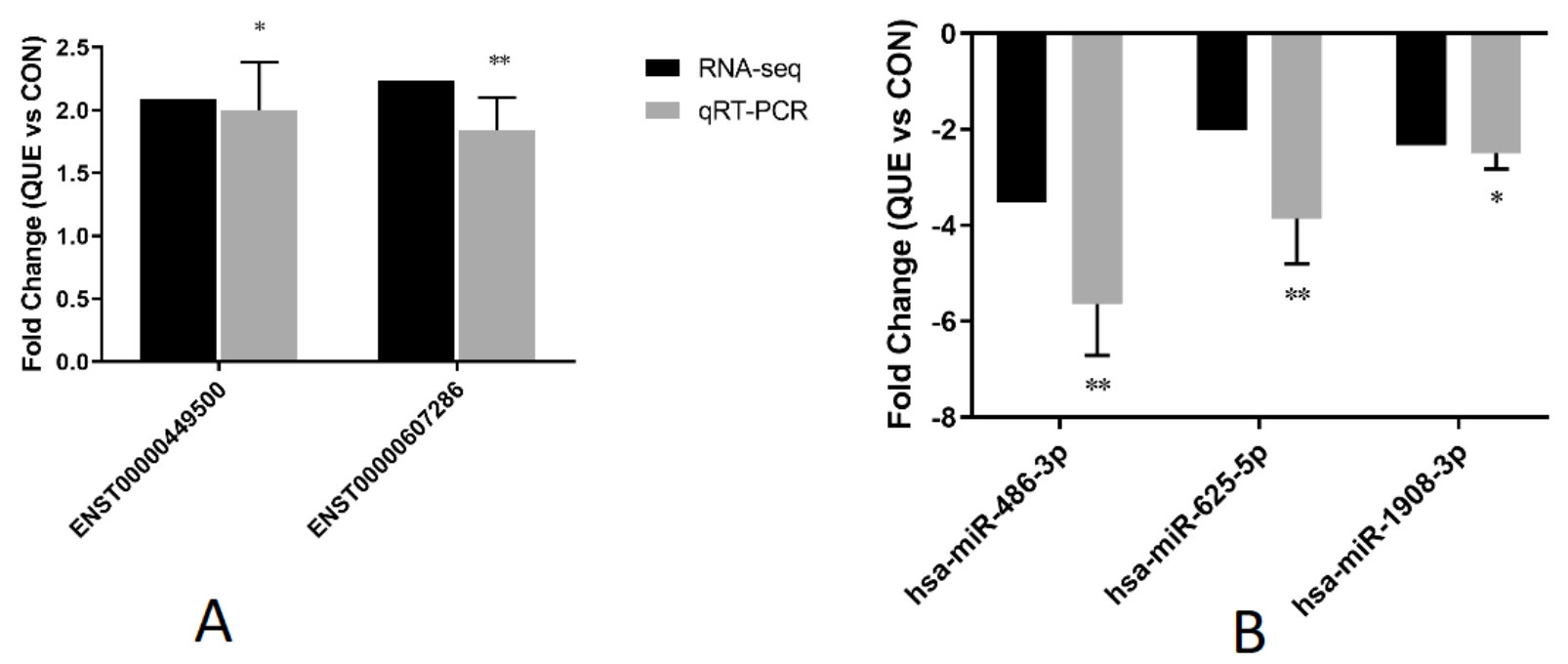

RNA-seq qRT-PCR

A

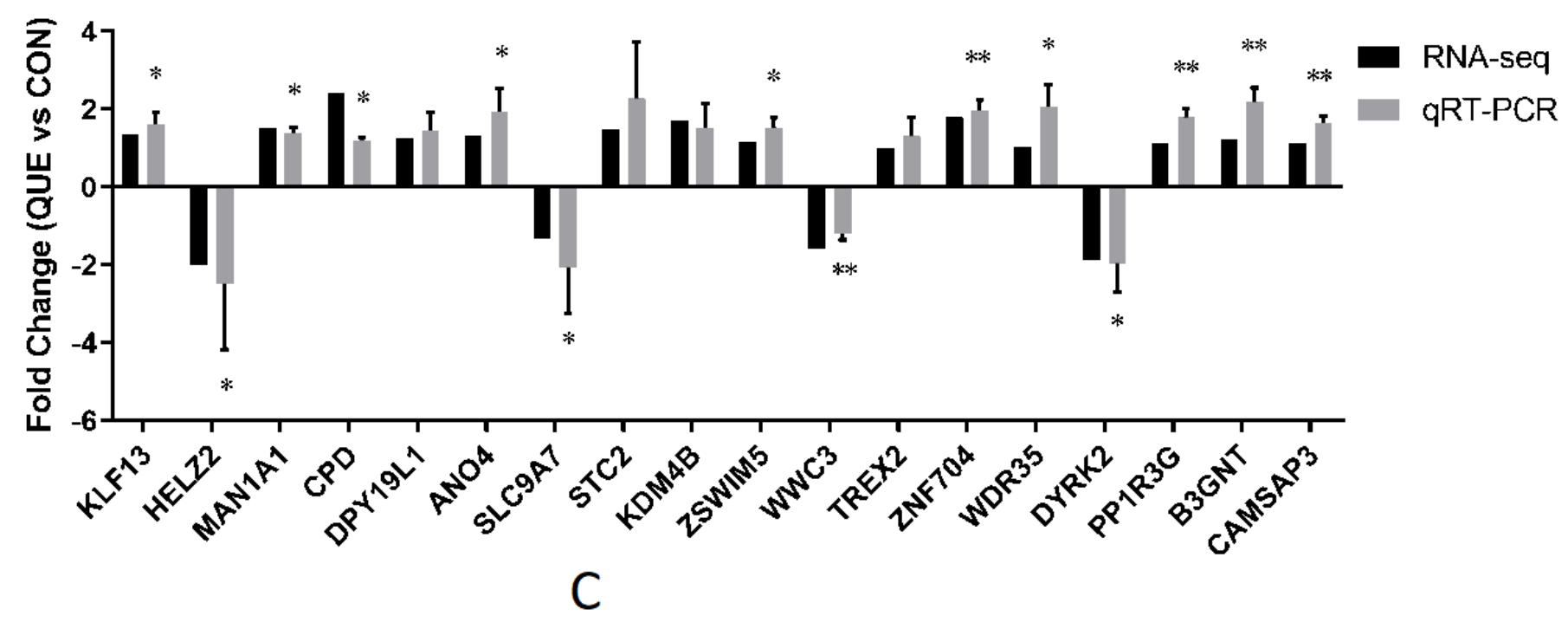

Figure 8

The qRT-PCR results of 2 DEIncRNAs (A), 3 DEmiRNAs (B), and 18 DEmRNAs (C) were compared with the sequencing results. The vertical axis represents the mean fold change (FC) (log2 scale) of each RNA measured by qRT-PCR or sequencing. ${ }^{*} P<0.05,{ }^{*} P<0.01$, compared with the control $(n=3)$. 